\title{
Aneurysmal subarachnoid hemorrhage: current concepts and updates
}

\author{
Hemorragia subaracnoidea aneurismática: conceitos atuais \\ Carolina ROUANET'1 , Gisele Sampaio SILVA'
}

\begin{abstract}
Aneurysmal subarachnoid hemorrhage is a condition with a considerable incidence variation worldwide. In Brazil, the exact epidemiology of aneurysmal SAH is unknown. The most common presenting symptom is headache, usually described as the worst headache ever felt. Head computed tomography, when performed within six hours of the ictus, has a sensitivity of nearly $100 \%$. It is important to classify the hemorrhage based on clinical and imaging features as a way to standardize communication. Classification also has prognostic value. In order to prevent rebleeding, there still is controversy regarding the ideal blood pressure levels and the use of antifibrinolytic therapy. The importance of definitely securing the aneurysm by endovascular coiling or surgical clipping cannot be overemphasized. Hydrocephalus, seizures, and intracranial pressure should also be managed. Delayed cerebral ischemia is a severe complication that should be prevented and treated aggressively. Systemic complications including cardiac and pulmonary issues, sodium abnormalities, fever, and thromboembolism frequently happen and may have na impact upon prognosis, requiring proper management.
\end{abstract}

Keywords: Subarachnoid hemorrhage; aneurysm; brain ischemia, vasospasm

\section{RESUMO}

Hemorragia subaracnoidea aneurismática (aHSA) é uma condição com grande variação de incidência mundialmente. No Brasil, não dispomos de números epidemiológicos exatos. A apresentação clínica mais comum da HSA é a cefaleia, usualmente descrita como a pior da vida. A tomografia de crânio, quando feita nas primeiras 6 horas do ictus, tem uma sensibilidade próxima a $100 \%$. É importante classificar a hemorragia utilizando escalas clínicas e radiológicas, como forma de padronizar a comunicação, e também predizer prognóstico. Para prevenção de ressangramento, ainda há controvérsias quanto aos níveis pressóricos ideais e uso de antifibrinolíticos. 0 tratamento definitivo do aneurisma, por sua vez, é fundamental, seja por meio endovascular ou cirúrgico. Hidrocefalia, crises epilépticas e a pressão intracraniana devem ser manejadas de forma apropriada. Isquemia cerebral tardia é uma complicação grave que deve ser prevenida e tratada agressivamente. Complicações sistêmicas, incluindo cardíacas, pulmonares, anormalidades de sódio, febre e tromboembolismo ocorrem frequentemente e podem ter impacto no prognóstico, necessitando de manejo adequado.

Palavras-chave: hemorragia subaracnoidea; aneurisma; isquemia encefálica; vasoespasmo

\section{EPIDEMIOLOGY}

Aneurysmal subarachnoid hemorrhage is a condition with considerable incidence variation worldwide. Finland is the country with the highest incidence, with 22.5 patients per 100,000 inhabitants. On the othe rhand, China has na incidence of $2 / 100,000$. The USA reports 14.5 patients per 100,000 population. However, as $15 \%-30 \%$ of deaths happen before hospital admission, the real incidence of aneurysmal SAH is probably higher' ${ }^{1}$. In Brazil, the exact epidemiology of aneurysmal SAH is unknown.
The estimated 30 -day mortality rate is $35 \%$, with great morbidity among the survivors (a third of whom require full care, and a third are not able to return to work).

In the past decades, mortality has been decreasing with the technical advances in medicine. A meta-analysis from 2009 has shown that between 1973 and 2002, there has been a $17 \%$ reduction in patient fatality ${ }^{2}$.

Risk factors for aneurysmal SAH include female sex, increasing age (with a peak around 50 years-old), smoking, hypertension, alcohol abuse, use of sympathomimetic drugs, familial history of aneurysms or SAH (more than two 
first-degree relatives), and some genetic conditions (autosomal dominant polycystic kidney disease and type IV EhlersDanlos syndrome) $)^{3}$.

\section{Clinical presentation and diagnosis}

The most common presenting symptom is headache, which is usually described as the worst headache ever felt, is abrupt and peaks in intensity in one hour at most ${ }^{4}$. Around $10-40 \%$ of patients have a warning leak or sentinel episode, which is a similar headache that precedes the bleeding by two to eight weeks. The presence of a sentinel episode increases the likelihood of death or disability four-fold 5 .

Nausea and vomiting happens in $77 \%$ of the cases, loss of consciousness in 53\%, meningismus in 35\%, focal deficits in $10 \%$, and Terson syndrome (vitreous hemorrhage associated with SAH) in $40 \%$ of patients p $^{3,4,5}$.

In patients with these symptoms, the threshold for na emergency physician to order a noncontrast brain computed tomography (CT) should be low. When performed within six hours of the ictus, it has a sensitivity of nearly $100 \%$. It falls to $93 \%$ after 24 hours and to less than $60 \%$ after five days ${ }^{4}$.

The traditional next step after a high suspicion of aneurysmal SAH and a negative CT has always been a lumbar puncture to look for blood or xanthochromia in the cerebrospinal fluid. Although it is still a class I, level of evidence B recommendation according to current guidelines, this approach is now a matter of debate. Some claim that since the incidence of $\mathrm{SAH}$ is low and it can be difficult to distinguish between
SAH and puncture-related trauma, lumbar puncture is no longer of great value ${ }^{5}$.

Another approach after a negative head CT is to perform a brain magnetic resonance imaging. Some sequences, such as fluid-attenuated inversion recovery, próton density, and gradient-echo are highly sensitive to blood.

A negative CT should not be enough to rule out SAH in high suspicion cases s $^{3,4,5}$.

In order to look for aneurysms $>3 \mathrm{~mm}$, CT angiography has a sensitivitythat approaches $100 \%$ and is comparable with the gold standard-digital subtraction angiography. The latter has the limitation of being na invasive and not universally-available method. More recently, CT angiography performed in 64-row multislice scanners and 320-detector row scanners has proven to have na excellent sensitivity and specificity for the detection of even small aneurysms $(<3 \mathrm{~mm})^{6}$.

\section{Clinical and radiological scales}

After the diagnosis of SAH, it is important to classify the hemorrhage based on clinical and imaging features. Classifications are important because they carry prognostic value and standardize communication. The strongest predictors of outcomes are the severity of neurological impairment on admission and the amount of blood in the subarachnoid space.

The most used clinical scales are the Hunt and Hess, and the World Federation of Neurological Surgeons scales (Table 1) 13.4. $^{3,4}$

Regarding radiological scales, the most used are the original Fisher Scale, and themodified Fisher Scale (Table 2).

Table 1.Clinical scales (World Federation of Neurological Surgeons and Hunt and Hess Scales).

\begin{tabular}{lcr}
\hline SAH grade & World Federation of Neurological Surgeons Scale & Hunt and hess scale \\
\hline 1 (good) & GCS 15, no motor deficit & Asymptomatic or mild headache and slight nuchal rigidity \\
2 (good) & GCS 14-13, no motor deficit & $\begin{array}{c}\text { Moderate to severe headache, nuchal rigidity, no focal } \\
\text { Neurologic deficit other than cranial nerve palsy }\end{array}$ \\
3 (good) & GCS 14-13, motor deficit & Confusion, lethargy, or mild focal neurologic deficit \\
4 (poor) & GCS 12-7, with or without motor deficit & Other than cranial nerve palsy \\
5 (poor) & GCS 6-3, with or without motor deficit & Stupor or moderate-to-severe hemiparesis
\end{tabular}

Table 2.Radiological Scales (Fisher and Modified Fished Scales).

\begin{tabular}{lcc}
\hline Grade & Original Fisher & Modified Fisher \\
\hline 0 & - & No SAH or IVH \\
1 & Diffuse, thin SAH, no clot $>1 \mathrm{~mm}$ in thickness \\
2 & Localized thick layer of subarachnoid & Minimum or thin SAH, no IVH in either lateral ventricle \\
3 & clot $>1 \mathrm{~mm}$ in thickness & Minimum or thin SAH, with IVH in both lateral ventricles \\
& Predominant IVH or intracerebral & Thick SAH, no IVH \\
\hline & hemorrhage without thick SAH & Thick SAH with IVH in both lateral ventricles
\end{tabular}


Table 3.Clinica land radiological scale (Vasograde).

\begin{tabular}{lcc} 
Vasograde & $\begin{array}{c}\text { World Federation of } \\
\text { Neurological Surgeons }\end{array}$ & Modified Fisher \\
\hline Green & $01 / 02$ & $01 / 02$ \\
Yellow & $01 / 03$ & $03 / 04$ \\
Red & $04 / 05$ & Any \\
\hline
\end{tabular}

The main goal of the radiological scales is to predict the risk of developing delayed cerebral ischemia (DCI)/vasospasm. However, in the original Fisher scale, a patient with grade 3 bleeding had more chances of developing DCI/vasospasm than a patient with grade 4.

According to the modified Fisher Scale, an SAH completely filling any cistern or fissure, and intraventricular blood in both lateral ventricles, has a higher risk of developing DCI. Not identified as a risk factor, intraparenchymal hemorrhage was left out of the new classification.

Summarizing, in the original Fisher Scale, when compared with grade 1 , grade 2 has na odds ratio (OR) of 1.3; grade 3 has an OR of 2.2; and grade 4 has an OR of 1.7 for developing DCI/vasospasm. In themodified Fisher Scale, when compared with grades $0 / 1$, grade 2 has an OR of 1.6; grade 3 has an OR of 1.6; and grade 4 has an OR of $2.2^{7,8}$.

The VASOGRADE scale combines clinical and radiological features (Table 3). It positively correlates with the risk of DCI. When compared with green, the yellow VASOGRADE has an OR of 1.31, and the red has an OR of 3.19 for developing $\mathrm{DCI} /$ vasospasm$^{9}$.

\section{PATHOPHYSIOLOGY}

When an aneurysm ruptures, blood pours into the subarachnoid space leading to a Sharp and sudden increase in intracranial pressure, jeopardizing cerebral perfusion pressure and leading to global ischemia. This is the postulated mechanism of the loss of consciousness that happens in about $50 \%$ of the patients. Also, the blood itself mechanically damages the brain, contributing to global ischemia. ${ }^{10,11}$

There is a massive sympathetic tone increase that starts to cause systemic complications (acute lung injury, neurogenic pulmonary edema, cardiac dysfunction) and a systemic inflammatory syndrome develops.

This series of events is part of what is called early brain injury, a process that starts Just after the aneurysmal rupture and is characterized by microcirculatory constriction, microthrombosis, disruption of the blood-brain barrier, vasogenic and cytotoxic edema, endothelia land neuronal death ${ }^{10,11}$

\section{Prevention of rebleeding}

After the diagnosis, the next step includes measures toavoid rebleeding. The incidence of rebleeding ranges from $7-22 \%$ in the first three days. In the first 24 hours, it is called ultra-early rebleeding, with na incidence of $15 \%$ and a mortality rate as high as $70 \%$. Patients with larger aneurysms and poor grade SAH are at higher risk ${ }^{4}$.

Whenever possible, patients should be transferred to a high-volume center, defined as at least $35 \mathrm{SAH}$, but ideally> $60 \mathrm{SAH}$ treatments per year. There is data showing that mortality is inversely proportional to the number of patients treated. A $24 \%$ reduction in mortality per year is estimated for each 100 patients treated. It is also important tohave a neurological intensive care unit and a multidisciplinary team ${ }^{1,12}$.

Management of blood pressure (BP) is na important part of the treatment, although there are no controlled, randomized trials regarding this topic. The American Heart Association guidelines establish that before the aneurysm is treated, systolic BP should remain below $160 \mathrm{mmHg}^{1}$. The European and theNeurocritical Care Society guidelines recommend a mean BP less than $110 \mathrm{mmHg}$, also taking into account premorbid baseline pressures ${ }^{13}$.

Ideally, drugs of choice for lowering BP should be titratable and short acting, such as nicardipine or labetolol. In Brazil, the most commonly used drug is sodium nitroprusside. After the aneurysm is secured, BP lowering medications should be withheld, unless there are signs of hypertensive crisis $^{1,4}$.

Antifibrinolytic therapy, which is a controversial topic, may play a role in the prevention of rebleeding. In some studies, antifibrinolytics were associated with good results. A Swedish study showed a decrease in rebleeding from $10.4 \%$ to $2.8 \%$ with the use of tranexamic acid $1 \mathrm{~g} 6 / 6$ hours, for a maximum of 72 hours. A Cochrane review, on the other hand, concluded that although antifibrinolytic therapy reduces rebleeding, this benefit is outweighed by the increase in ischemic events ${ }^{1,4,13}$.

According to the guidelines, when there is na unavoidable delay in securing the aneurysm and no contraindications, na early short-course of antifibrinolytics may be reasonable ${ }^{1,13}$.

There is an ongoing randomized, multicentric trial that Will possibly answer this question. It is testing $1 \mathrm{~g}$ of tranexamic acid Just after the diagnosis of SAH followed by continuous infusion of $1 \mathrm{~g}$ per eight hours to a maximum of 24 hours ( $4 \mathrm{~g}$ maximum), against placebo ${ }^{14}$.

The most effective way to prevent rebleeding, however, is to definitively secure the aneurysm, which can be achieved by endovascular coiling or surgical clipping.

Two randomized trials have compared bothstrategies, the International Subarachnoid Aneurysm Trial and the Barrow Ruptured Aneurysm Trial. With a follow-up of 18 years, the former trial showed a $23 \%$ relative reduction of the risk of death or dependence in patients who underwent coiling instead of clipping (number needed to treat of 14). However, obliteration rates were higher in the surgical group. It should be emphasized that the aneurysms included were considered appropriate for both methods of treatment, and were, in their majority, small and from the anterior circulation ${ }^{15}$. 
The Barrow Ruptured Aneurysm Trial had a follow-up of six years and showed no relevant differences between coiling and clipping for anterior circulation aneurysms. Regarding posterior circulation aneurysms, endovascular treatment was superior, although obliteration rates were lower ${ }^{16}$.

Recently, a new study was published with contrasting resultscompared with the trials mentioned above. It was a controlled, non randomized study that used na administrative dataset of more than 8,000 patients from Europe, Australia, and the USA, and compared outcomes after endovascular coiling and surgical clipping in aneurysmal SAH. In a multivariable logistic regression, the OR for 14-day patient fatality after coiling compared to clipping was 1.7 (95\% CI 1.1-2.7); for 90-day patient fatality, 1.28 (95\% CI 0.91-1.82); and for 90-day poor functional outcome, 0.78 (95\% CI 0.6-1.01). The conclusion was that, in clinical practice, endovascular treatment was associated with a higher 14-day patient fatality and nonsuperior outcomes at 90 days $^{17}$. A severe limitation of this analysis is that there was likely indication bias. It is possible that patients with the worst clinical condition were those treated by endovascular methods.

In summary, it is still unclearwho are the best candidates for clipping or coiling. Patient characteristics, topography and size of the aneurysm, as well as treatment expertise in treating brain aneurysms, should all be taken into account.

For aneurysms considered technically suitable for both methods, coiling may be preferred especially for those in the posterior circulation (particularly at the basilar artery apex), in poor grade $\mathrm{SAH}$, and in elderly patients.

For patients with large hematomas, with middle cerebral artery aneurysms, or those in whom bypass revascularization is deemed necessary, surgical clipping may be a better option, as well as for those who are Young and may benefit from a method with higher durability ${ }^{1,5}$.

There is also some debate regarding the Best moment to perform the procedure - early (within 72 hours) orultra-early (within 24 hours). The formal recommendation is to perform as early as is feasible ${ }^{1}$.

A retrospective, non randomized trial analyzed treatment $<24$ hours versus 24-72 hours afterthe ictus and concluded that it can be safely performed in the day time, in up to 72 hours, instead of as an emergency ${ }^{18}$.

There is na ongoing randomized, single-center Chinese study comparing early surgery (up to three days), intermediate surgery (four to seven days) and late surgery (more than seven days) in patients with poor grade $\mathrm{SAH}^{19}$.

\section{Hydrocephalus}

Hydrocephalus can occur in acute (our focus here) or chronic stages, in up to $50 \%$ of patients after SAH. When it leads to decreased level of consciousness, it requires na external ventricular drain. Up to $30 \%$ of those who present with poor grade SAH may become a good grade after drainage ${ }^{1,4}$.

Lumbar drainage may also be anoption; however, large hematomas and obstructive hydrocephalus are contraindications ${ }^{5}$.

There is some concern about the theoretical risk of rebleeding when the aneurysm is not yet treated (due to the increase of transmural pressure caused by fluid removal). Some studies claim that drainage is $\mathrm{safe}^{20}$, and some claim that it increases re-rupturerisk. A recent meta-analysis and systematic review found na association between drainage and rebleeding, with an OR of 3.92 (18.5\% of rebleeding in the external ventricular drain group versus $6.4 \%$ of rebleeding in the group that did not need na external ventricular drain), with a median time of one hour from the insertion of the drain until the rebleed ${ }^{21}$.

Another controversy is whether to maintain a continuous or intermittent drainage and how to wean the external ventricular drain or lumbar drain. What is usually warranted is to perform intermittent cerebrospinal fluid drainage and attempt na early clamp trial ${ }^{22}$.

Some studies claim that rapid weaning (immediate closure and observation for 24 hours) leads to reduced days of intensive care unit stay and the same rate of permanent shunts, compared with gradual weaning (over 96 hours with daily step-up followed by closure after 24 hours) ${ }^{23}$.

Multicenter prospective studies regarding the best management of the external ventricular drain are, therefore, warranted.

\section{Seizures}

Seizures can occur when the aneurysm ruptures, perioperatively (usually represents a re-rupture) or later (beyond the scope of this review) and their incidence ranges between 4-26\%.

Guidelines state that a short course (3-7 days) of seizure prophylaxis may be used in the immediate postbleeding period; however, this has a low level of evidence. Phenytoin should be avoided due to its effects on cognitive outcomes, incidence of vasospasm, and CT infarcts. The effects of other antiepileptic drugs are less clear. Levetiracetam is increasingly being used and seems to be a good option ${ }^{1,13}$.

\section{Increased intracranial pressure}

High intracranial pressure is a frequentearly complication that may develop due tobrain edema, intraparenchymal hemorrhage, hydrocephalus, or rebleeding. Intracranial pressure should be aggressively treated with head of bed elevation, sedation, analgesia, hyperosmolar therapy, normal ventilation (short periods of hyperventilation only as a bridge to therapy), cerebrospinal fluid drainage and, in refractory cases, barbiturate sedation, decompressive craniectomy, or hypothermia ${ }^{4,10}$.Most of the recommendations 
for the treatment of elevated intracranial pressure come from traumatic brain injury.

\section{Delayed cerebral ischemia: definition and pathophysiology}

Delayed cerebral ischemia occurs in upto $30 \%$ of the patients after SAH, usually within the first two weeks after the bleeding. It is defined as the occurrence of any focal neurological impairment or decrease of at leas ttwo points on the Glasgow Coma Scale, that lasts for at least one hour, is not apparent immediately after the aneurysm occlusion and cannot be attributed to other causes (hydrocephalus, infection, electrolyte disturbance, or seizure).

The risk of DCI is correlated mainly with the amount of cisterna land intraventricular blood on initia lneuroimaging (as discussed previously in the Fisher/modified Fisher scale). The risk is also higher in patients with a poor neurological examination post-resuscitation (as discussed previously in the World Federation of Neurological Surgeons/Hunt and Hess scales).

Traditionally, DCI has been related to arterial vasospasm. However, more recent evidence suggest that it has a complex pathophysiology with multiple postulated mechanisms, even occurring in territories without spasm ${ }^{10,11,25}$.

Currently, DCI is attributed to arterial vasospasm, microthrombosis, inflammation, microcirculation dysfunction and cortical spreading depolarization/ischemia.

Arterial vasospasm happens in $70 \%$ of the patients between days 3-14 post-bleeding. Its severity is related to the amount, thickness and persistence of blood.

Its main driver is the release of hemoglobin contents, leading to inflammation, oxygenfree radical reactions, endothelial injury, increase in the expression of endothelin 1, and reduction in nitric oxide. Some studies show cerebral infarction in $3 \%, 10 \%$, and $46 \%$ of patients with no/mild, moderate or severe vasospasm, respectively. Not only the spasm itself and its severity, but collateral bloodflow, brain metabolic demand, and BP levels also play a role in $\mathrm{DCI}^{11}$.

As Will bediscussed later, some drugs have been able to reduce arterial spasm but not DCI, and others were able to reduce DCI and poor outcomes, but with no change in vasospasm.

Microthrombosis seems to be abundant in regions correlated with vasospasm and DCI. The presence of blood and its products activate inflammatory pathways and lead to endothelial cell activation and damage. There is breakdown of the blood vessel basal lamina, destruction of collagen type IV, and hyperactivation of matrix metalloproteinase 9 , which together cause mural thrombus formationand release of microemboli.

A large arterial spasm also injures the endothelium, leading to platelet adherence, coagulation and propagation of the microemboli formation. The clotting processis fundamental to stopping the bleeding but generates a self-perpetuated prothrombotic mechanism. Some studies correlate a high level of von Willebrand factor with the occurrence of DCI ${ }^{11,25}$.

Inflammation also plays a major role in DCI. In SAH, there are inflammation-related genes with increased mRNA expression, upregulation of genes encoding membrane receptors, extracellular matrix components, cell adhesion molecules and proapoptotic proteins. With the rupture of the blood-brain barrier, inflammatory cells infiltrate the clot and the arteries, leading to increased expression of inflammatory cytokines, endothelin 1 , reactive oxygen species, and trigger oxidative stress.

In summary, after SAH there is a massive expression of proinflammatory cytokines, an invasion of inflammatory cells, and a proinflammatory state develops, which seems to contribute to arterial spasm and DCI ${ }^{11,25}$.

It is likely that microvascular constriction and dysfunction also play a role in the development of DCI. After SAH, at first there is pial arteriole constriction caused by altered vascular reactivity due to endothelial injury. Arteriolar smooth muscle is dysfunctional and the presence of blood increases vasoconstriction ${ }^{11}$.

Cortical spreading depolarization is a Gray matter depolarization wave that propagates at a speed of 2-5 $\mathrm{mm}$ per minute, leading todepression in brain electrical potential (spreading depolarization) and electrical activity (spreading depression). Cortical arterioles usually respond to depolarization with vasodilation and hyperemia. However, after SAH, vasoconstriction may occur, leading to waves of cortical hypoperfusion (cortical spreading ischemia) and brain damage.

The presence of elevated potassium concentrations, the reduced levels of nitric oxide and other metabolic changes favor the occurrence of cortical spreading depolarization. Some studies temporally and spatially correlate cortical spreading depolarization with DCI. Depolarizations that last longer than 60 minutes may be strongly correlated with infarction ${ }^{10,11,25}$.

\section{Delayed cerebral ischemia: prevention and monitoring}

In order to prevent DCI, the only pharmacologicallyapproved therapy is nimodipine $60 \mathrm{mg}$ every four hours for 21 days. It has been shown to reduce cerebral infarction, poor outcome and death, but not to reduce angiographic spasm ${ }^{24}$. If hypotension occurs, the dose can be divided in $30 \mathrm{mg}$ every two hours or reduced to $30 \mathrm{mg}$ every four hours. This recommendation is a class I, level of evidence A, according to current guidelines ${ }^{1,13}$.

Hyponatremia and hypovolemia frequently occur after $\mathrm{SAH}$, are associated with DCI, and should be avoided. The target is normal serum sodium and euvolemia, which can be achieved by isotonic crystalloid fluid resuscitation. If needed, fludrocortisone may be an option. 
The previous classic "triple $\mathrm{H}$ therapy" (hypertension, hemodilution, andhypervolemia) is no longer recommended as there is no evidence that it improves neurological outcomes and it can increase the risk of cardiac dysfunction, pulmonary edema, and infection.

There have been multiple failed attempts to prevent DCI by targeting its complex pathophysiology. Clinical trials with magnesium, clazosentan (antagonist of endothelin 1 receptor), aspirin, heparin, erythropoietin, nicardipine, methylprednisolone, prophylactic use of balloon angioplasty, intraarterial or intravenous milrinone, and even with intrathecal drugs, have all been unsuccessfull to date. Consequently, none of these therapies are formally recommended ${ }^{4,25}$.

Delayed cerebral ischemia is considered a reversible condition IF treated promptly. Monitoring, recognition, and aggressive treatment are fundamental. Among those patients who are awake, frequent neurological examination is the gold standard for detecting DCI. However, in those with poor grade $\mathrm{SAH}$, its detection is frequently challenging due to the decreased level of consciousness or the use of sedation, leading to the need of advanced monitoring strategies ${ }^{4,25}$.

Transcranial Doppler is a non invasive test that allows indirect evaluation of proximal large vessel cerebral blood flow based on bloodvelocities. It can be performed at the bedside and be repeated whenever needed. Middle cerebral artery mean velocities $<120 \mathrm{~cm} / \mathrm{s}$ have a high negative predictive value for vasospasm, and velocities $>180 \mathrm{~cm} / \mathrm{s}$ or 200 $\mathrm{cm} / \mathrm{s}$ have a high positive predictive value. The Lindegaard Index is defined by themiddle cerebral artery mean cerebral blood flow velocity divided by the extracranial internal carotid artery mean cerebral blood flow velocity, and has fewer systemic hemodynamic variations. When it is $>3$, the index indicates vasospasm, and $>6$ indicates severe vasospasm. Serial examinations are alsoimportant as an increase of $50 \mathrm{~cm} / \mathrm{s}$ in 24 hours is also a "red flag",, 25

Sensitivity and specificity of transcranial Doppler for vasospasm diagnosis varies widely between authors, ranging from $39-94 \%$ and $70-100 \%$, respectively ${ }^{26}$. It is considered by the American Heart Association/American Stroke Association guideline as class IIa, level of evidence B, and by theNeurocritical Care Society guideline as having a moderate quality of evidence, with strong recommendation ${ }^{1,13}$.

Continuous electroencephalography (EEG) is a useful method for the detection of DCI and has the advantages of being non invasive, monitoring broad regions of the brain and detecting epileptiform discharges and electrographic crises. In the presence of cortical spreading depolarization and cortical ischemia, EEG changes may precede the occurrence of symptoms. The most sensitive EEG patterns for the prediction of DCI are reduction in the alpha/delta ratio and decrease of alpha variability ${ }^{10,25}$. The presence of interictal discharges, especially when starting several days after the bleeding, may also be correlated with na increased risk of $\mathrm{DCI}^{27}$.
Computedtomographic (CT) perfusion is na appealing method since it allows for the evaluation of spasm of large and small vessels and their functional consequences on cerebral bloodflow. The most accepted cutoff values are a mean transit time $>5-6.5$ seconds and/or regional cerebral blood flow $<25-40 \mathrm{ml} / 100 \mathrm{~g} / \mathrm{min}^{6,10}$.

One meta-analysis concluded that SAH patients with an abnormal CT perfusion are 23 times more likely to develop DCI, with a sensitivity of $84 \%$ and specificity of $77 \%$. Another meta-analysis showed that na increase in mean transit time during hospitalization of 1.5 secondsor more, or a reduction of cerebral blood flow of $11.9 \mathrm{~mL} / 100 \mathrm{~g} / \mathrm{min}$, was diagnostic for $\mathrm{DCl}^{28,29}$.

Monitoring brain tissue partial pressure oxygenation is na invasive, direct method that measures continuous but regional levels of oxygen, and may detect early changes in brain oxygenation, preceding ischemic damage and clinical manifestations. Levels $<20 \mathrm{mmHg}$ are a warning sign, and< $15 \mathrm{mmHg}$ require immediate intervention.

Cerebral microdialysis measures interstitial levels of glucose, lactate, pyruvate and glutamate. The most-studied markers of anaerobic metabolism and thus indicators of cerebral ischemia are the lactate-pyruvate ratio and glucose levels. A lactate-pyruvate ratio $>40$ and/or glucose $<0.5 \mathrm{mM}$ may be indicators of DCI.

Partial pressure oxygenation and cerebral microdialysis have proven to be more accurate than the classic evaluation by intracranial pressure and cerebral perfusion pressure, as hypoxia and a cellular energy crisis may occur even with normal intracranial pressure and cerebral perfusion pressure ${ }^{10}$.

\section{Delayed cerebral ischemia: treatment}

The first step of DCI treatment is usually a normal saline bolus $(15 \mathrm{~mL} / \mathrm{kg})$ in order to increase cerebral blood flow to ischemic areas and maintain euvolemia and normal cerebral blood volume.

Triple $\mathrm{H}$ therapy is not recommended as part of DCI treatment as it leads to high complication rates and has not proven to be beneficial. Hypervolemia alone has proven to be ineffective and detrimental, raising the chances of pulmonary and cerebral edema, infections, coagulopathies, prolonged ventilator weaning, and longer intensive care unit stay. Hemodilution may reduce oxygen delivery and raise cerebral ischemic volume.

Normovolemic-induced hypertension using vasopressors, however, remains a cornerstone of first line treatment for DCI. The aim is to improve cerebral blood flow and cerebral oxygenation, and clinical response may be seen in up to $70 \%$ of the patients. Norepinephrine, dopamine or phenylephrine may be used, depending on the clinical scenario. Nowadays, the recommendation is to induce hypertension unless the patient is already hypertensive or there is a formal contraindication. 
Blood pressure should be raised in a stepwise fashion and titrated to clinical response or improvement of multimodal parameters. Lack of response in 30 minutes should trigger escalation of BP targets. Most centers use a maximal target of systolic BP $220 \mathrm{mmHg}$, mean arterial pressure $140 \mathrm{mmHg}$ or cerebral perfusion pressure $120 \mathrm{mmHg}$. Once there is resolution of DCI, the therapy is maintained for 24-48 hours of stable symptoms, and then slowly withdrawn ${ }^{10,11,25}$.

The Hypertension Induction in the Management of Aneurysmal Subarachnoid Haemorrhage with Secondary Ischaemia study was the first randomized trial aiming to evaluate induced hypertension as a treatment for $\mathrm{DCl}^{30}$. It was prematurely interrupted with 41 patients (the original design aimed to include 240 patients) due to lack of effect on cerebral perfusion and slow recruitment. The group with induced hypertension had an adjusted risk ratio for poor outcome of 1.0 (95\% CI 0.6-1.8) and risk for serious events 2.1 (95\% CI 0.9-5.0). Due to the premature interruption, it was underpowered to support induced hypertension as a therapy for DCI. It demonstrated, however, that serious adverse effects may happen with elevated BP. There are several criticisms of the study, such as the small number of patients (only 21 in the hypertension group), possibly an insufficient target of BP and the lack of a better selection of patients ${ }^{30}$. New trials addressing this topic are still needed.

After hypertension, the next step in the treatmentof DCI (tier two) may be cardiac output augmentation with dobutamine or milrinone, attempting to improve brain perfusion. Milrinone is a selective inhibitor of phosphodiesterase type III isoenzyme with inotropic and vasodilator properties. It has a short halflife (around 50 minutes) - the reason why it is used as continuous infusion ${ }^{13,25}$.

The Montreal Neurological Hospital published their protocol in $2012^{31}$, with extensive experience with the drug. Their protocol consisted of a bolus of $0.1-0.2 \mathrm{mg} / \mathrm{kg}$ intravenous milrinone, followed by a continuous intravenous infusion of $0.75 \mathrm{mcg} / \mathrm{kg} / \mathrm{min}-1.25 \mathrm{mcg} / \mathrm{kg} / \mathrm{min}$. If there is hypotension, vasopressors should be initiated, aiming for a mean arterial pressure of $90 \mathrm{mmHg}$. The infusion should be maintained for 72 hours of stable symptoms and then weaning is initiated, decreasing $0.25 \mathrm{mcg} / \mathrm{kg} / \mathrm{min}$ every $28-48$ hours. If symptoms recur, the protocol should be restarted. A total of 88 patients were followed, five deaths occurred and $48.9 \%$ of the surviving patients returned to their basal condition and $75 \%$ had a modified Rankin scale $\leq 2$. No significant collateral effects were reported ${ }^{31}$.

A systematic review showed that the literature is still very poor regarding milrinone and DCI, with no randomized or case controlled studies published, leading to very low level of evidence regarding this topic ${ }^{32}$.

Recently, a randomized controlled study that aimed to compare milrinone plus hyperdynamic therapy was stopped due to lack of eligible subjects ${ }^{33}$.
If medical therapy fails, the next step is endovascular therapy (mechanical dilation with balloon angioplasty and/ or pharmacological dilation with intra-arterial drugs).

Balloon angioplasty appears safe, with reported neurological improvement rates as high as $90 \%$. The mechanism of action is relatedto the stretching and disruption of degenerative muscle and proliferative components in the media of spastic arteries. It is limited to proximal vessels. Potential complications are vessel perforation, reperfusion injury, distal embolism and thrombosis. Recurrence is rare.

Intra-arterial therapy has the advantages of distal penetration, and is easier and safer to perform. It has a transitory effect (depending on the half life of the drug), and recurrence often hpens. There is also a theoretical risk of increased intracranial pressure secondary to vasodilation and systemic hypotension. Nowadays, the most used drugs are nicardipine, verapamil and milrinone $\mathrm{e}^{25,34}$.

A recent meta-analysis of 55 studies on intra-arterial therapy reported $89 \%$ angiographic improvement, 57\% neurological improvement, $66 \%$ good clinical outcome and $9 \%$ deaths ${ }^{35}$.

Other interventions such as intrathecal drugs (milrinone, sodium nitroprusside, nicardipine), aortic flow diversion, intra-aortic balloon pump counter-pulsation are experimental strategies under investigation.

\section{SYSTEMIC COMPLICATIONS}

Sodium and water abnormalities are common after SAH, with hyponatremia occurring in 30-50\% of patients, hypovolemia in $17-30 \%$, both being associated with negative outcomes. The pathophysiology is not completely understood, but increased natriuretic peptide concentrations, sympathetic nervous system hyperactivation and hyperreninemic hypoaldosteronism may contribute.

Hyponatremia may be due to the syndrome of inappropriate antidiuretic hormone (normal intravascular volume) or cerebral salt wasting syndrome (depleted intravascular volume).

Water restriction should bea avoided in treating hyponatremia due to the risk of developing DCI. Hypertonic fluids and fludrocortisone are better options ${ }^{1,4}$. Especially in poor grade patients, early intensive hemodynamic monitoring may improve outcomes ${ }^{36}$.

Cardiac complications are related to sympathetic hyperactivity and catecholamine-induced myocardial dysfunction, and are also associated with poor outcomes. Positive cardiac enzymes, electrographic changes, arrhythmias, motion wall abnormalities, stunned myocardium and cardiogenic shockmay occur 4 .

A meta-analysis demonstrated that motion wall abnormalities, increased cardiac enzymes, increased brain natriuretic peptide, Q and T waves and ST segment abnormalities were associated with worse outcomes, 
increased mortality and increased the occurrenceof $\mathrm{DCI}^{37}$. Treatment is mainly supportive.

Pulmonary complications occur in 20-30\% of patients with a positive association of DCI with death. Pneumonia, acute respiratory distress syndrome, cardiogenic or neurogenic pulmonary edema and pulmonary embolism may occur ${ }^{4}$.

Fever is themost common medical complication after SAH and should be aggressively treated, aiming for normothermia. It can be infectious or noninfectious, with the latter being a diagnosis of exclusion ${ }^{1,4,13}$.

Glucose management is of major importance. Hypo- and hyperglycemia are correlated with an energetic crisis and worse prognosis. Serum glucose levels $80-180 \mathrm{mg} / \mathrm{dl}$ seem to be reasonable ${ }^{1,4,13}$.
Prevention of deep venous thrombosis should be practiced in all SAH patients. Before the aneurysm is safe, compression devices must be used. Chemical prophylaxis can be started 24 hours after surgery ${ }^{1,13}$.

\section{CONCLUSION}

In conclusion, $\mathrm{SAH}$ is a disease with high morbimortality, whose treatment goes through several distinct phases ranging from the imminent risk of rebleeding to late cerebral ischemia. The approach in an intensive care setting is crucial to minimize the risks of complications and to optimize the clinical prognosis of patients with SAH.

\section{References}

1. Connolly ES Jr, Rabinstein AA, Carhuapoma JR, Derdeyn CP, Dion J, Higashida RT, et al. Guidelines for the management of aneurysmal subarachnoid hemorrhage: a guideline for healthcare professionals from the American Heart Association/ American Stroke Association. Stroke. 2012 Jun;43(6):1711-37. https://doi.org/10.1161/STR.0b013e3182587839

2. Nieuwkamp DJ, Setz LE, Algra A, Linn FH, de Rooij NK, Rinkel GJ. Changes in case fatality of aneurysmal subarachnoid haemorrhage over time, according to age, sex, and region: a meta-analysis. Lancet Neurol. 2009 Jul;8(7):635-42. https://doi.org/10.1016/S1474-4422(09)70126-7

3. Suarez JI. Suarez. Diagnosis and management of subarachnoid hemorrhage. Continuum (MinneapMinn). 2015 Oct;21(5 NeurocriticalCare):1263-87. https://doi.org/10.1212/CON.0000000000000217

4. Manoel ALO, Turkel-Parrella D, Duggal A, Murphy A, McCredie V, Marotta TR. Managing aneurysmal subarachnoid hemorrhage: it takes a team. CleveClin J Med. 2015 Mar;82(3):177-92. https://doi.org/10.3949/ccjm.82a.14021

5. Lawton MT, Vates GE. Subarachnoid Hemorrhage. N Engl J Med. 2017 Jul;377(3):257-66. https://doi.org/10.1056/NEJMcp1605827

6. Manoel ALO, Mansur A, Murphy A, Turkel-Parrella D, Macdonald $\mathrm{M}$, Macdonald RL, et al. Aneurysmal subarachnoid hemorrhage from a neuroimaging perspective. CritCare. 2014 Nov 13;18(6):557. https://doi.org/10.1186/s13054-014-0557-2

7. Claassen J, Bernardini GL, Kreiter K, Bates J, Du YE, Copeland $D$, et al. Effect of cisternal and ventricular blood on risk of delayed cerebral ischemia after subarachnoid hemorrhage: the Fisher scale revisited. Stroke. 2001 Sep;32(9):2012-20. https://doi.org/10.1161/hs0901.095677

8. Frontera JA, Claassen J, Schmidt JM, Wartenberg KE, Temes R, Connolly ES Jr, et al. Prediction of symptomatic vasospasm after subarachnoid hemorrhage: the modified fisher scale. Neurosurgery. $2006 \mathrm{Jul} ; 59(1): 21-7$. https://doi.org/10.1227/01.NEU.0000218821.34014.1B

9. Manoel ALO, Jaja BN, Germans MR, Yan H, Qian W, Kouzmina E, et al..The Vasograde: a simples grading scale for prediction of delayed cerebral ischemia after subarachnoid hemorrhage. Stroke. 2015;46(7):1826-31. https://doi.org/10.1161/STROKEAHA.115.008728

10. Airton LO. Manoel, Alberto Goffi, Tom R Marotta. The critical care management of poor-grade subarachnoid hemorrhage. CritCare. 2016;20:1-19. https://doi.org/10.1186/s13054-016-1193-9
11. Macdonald RL. Delayed neurological deterioration after subarachnoid hemorrhage. Nature. 2013;246:44-59.

12. Prabhakaran S, Fonarow GC, Smith EE, Liang L, Xian Y, Neely M, et al. Hospital case volume is associated with mortality in patients hospitalized with subarachnoid hemorrhage. Neurosurgery. $2014 \mathrm{Nov}$;75(5):500-8. https://doi.org/10.1227/NEU.00000000000000475

13. Diringer MN, Bleck TP, Claude Hemphill J 3rd, Menon D, Shutter $L$, Vespa P, et al. Critical care management of patients following aneurysmal subarachnoid hemorrhage: recommendations from the Neurocritical care Society's Multidisciplinary Consensus Conference. NeurocritCare. 2011 Sep;15(2):211-40. https://doi.org/10.1007/s12028-011-9605-9

14. Germans MR, Post R, Coert BA, Rinkel GJ, Vandertop WP, Verbaan D. Ultra-early tranexamic acid after subarachnoid hemorrhage (ULTRA): study protocol for a randomized controlled trial. Trials. 2013 May;14(1):143. https://doi.org/10.1186/1745-6215-14-143

15. Molyneux AJ, Birks J, Clarke A, Sneade M, Kerr RS. The durability of endovascular coiling versus neurosurgical clipping of ruptured cerebral aneurysms: 18 year follow -up of the UK cohort of the International Subarachnoid HemorrhageTrial (ISAT). Lancet. 2015 Feb 21;385(9969):691-7. https://10.1016/S0140-6736(14)60975-2

16. Robert F Spetzler, Cameron G MacDougall, Joseph M Zabramski et al. The barrow ruptured aneurysm trial: 6-years results. J Neurosurg. 2015 Sep;123(3):609-17. https://doi.org/10.3171/2014.9.JNS141749

17. Lindgren A, Turner EB, Sillekens T, Meretoja A, Lee JM,Hemmen TM, et al. Outcome after clipping and coiling for aneurysmal subarachnoid hemorrhage in clinical practice in Europe, USA, and Australia. Neurosurgery. 2019 May 1;84(5):1019-27. https://doi.org/10.1093/neuros/nyy223

18. Oudshoorn SC, Rinkel GJ, Molyneux AJ, Kerr RS, DorhoutMees SM, Backes D, et al. Aneurysm treatment $<24$ versus $24-72 \mathrm{~h}$ after subarachnoid hemorrhage. NeurocritCare. 2014 Aug;21(1):4-13. https://doi.org/10.1007/s12028-014-9969-8

19. Zhang Q, Ma L, Liu Y, He M, Sun H, Wang X, et al. Timing ofoperation for poor-grade aneurysmal subarachnoid hemorrhage: study protocol for a randomized controlled trial. BMC Neurol. 2013 Aug;13(1):108. https://doi.org/10.1186/1471-2377-13-108

20. Hellingman CA, Bergh WM, Beijer IS, Dijk GW, Algra A, Gijn J, et al. Risk of rebleeding after treatment of acute hydrocephalus in patients with aneurysmal subarachnoid hemorrhage. Stroke. 2007 Jan;38(1):96-9. https://doi.org/10.1161/01.STR.0000251841.51332.1d 
21. Cagnazzo F, Gambacciani C, Morganti R, Perrini P. Aneurysm rebleeding after placement of external ventricular drainage: a systematic review and meta-analysis. Acta Neurochir (Wien). 2017 Apr;159(4):695-704. https://doi.org/10.1007/s00701-017-3124-1

22. Chung DY, Mayer SA, Rordorf GA. Chung, Stephan A Mayer, Guy A Rordorf. External ventricular drains after subarachnoid hemorrhage: is less more? NeurocritCare. 2018 Apr;28(2):157-61. https://doi.org/10.1007/s12028-017-0443-2

23. Klopfenstein JD, Kim LJ, Feiz-Erfan I, Hott JS, Goslar P, Zabramski JM, et al. Comparison of rapid and gradual weaning from external ventricular drainage in patients with aneurysmal subarachnoid hemorrhage: a prospective randomized trial.J Neurosurg. 2004 Feb;100(2):225-9. https://doi.org/10.3171/jns.2004.100.2.0225

24. Petruk KC, West M, Mohr G, Weir BK, Benoit BG, Gentili F, et al.Nimodipine treatment in poor-grade aneurysm patients. Results of a multicenter double-blind placebo-controlled trial. J Neurosurg. 1988 Apr;68(4):505-17. https://doi.org/10.3171/jns.1988.68.4.0505

25. Francoeur CL, Mayer SA. Management of delayed cerebral ischemia ater subarachnoid hemorrhage. CritCare. 2016;20:277-89. https://doi.org/10.1186/s13054-016-1447-6

26. Sloan MA, Alexandrov AV, Tegeler $\mathrm{CH}$, Spencer MP, Caplan LR, Feldmann E, et al. Assessment: transcranial Doppler ultrasonography: reports of the therapeutics and technology assessment subcommittee of the American Academy of Neurology. Neurology. 2004 May;62(9):1468-81. https://doi.org/10.1212/WNL.62.9.1468

27. Kim JÁ, Rosenthal ES, Biswal S, Zafar S, Shenoy AV, O'Connor KL, et al. Epileptiform abnormalities predict delayed cerebral ischemia in subarachnoid hemorrhage. ClinNeurophysiol. 2017 Jun;128(6):10919. https://doi.org/10.1016/j.clinph.2017.01.016

28. Cremers CH, Schaaf IC, Wensink E, Greving JP, Rinkel GJ, Velthuis BK, et al. CT perfusion and delayed cerebral ischemia in aneurysmal subarachnoid hemorrhage: a systematic review and meta-analysis. J CerebBloodFlowMetab. 2014 Feb;34(2):200-7. https://doi.org/10.1038/jcbfm.2013.208

29. Mir DI, Gupta A, Dunning A, Puchi L, Robinson CL, Epstein HA, et al. CT perfusion for detection of delayed cerebral ischemia in aneurysmal subarachnoid hemorrhage: a systematic review and meta-analysis. AJNR Am J Neuroradiol. 2014 May;35(5):866-71. https://doi.org/10.3174/ajnr.A3787

30. Gathier CS, Bergh WM, Jagt M, Verweij BH, Dankbaar JW, Müller MC, et al. Induced hypertension for delayed cerebral ischemia after aneurysmal subarachnoid hemorrhage: a randomized clinical trial. Stroke. 2018 Jan;49(1):76-83. https://doi.org/10.1161/STROKEAHA.117.017956

31. Lannes M, Teitelbaum J, Pilar Cortés M, Cardoso M, Angle M. Milrinone and homeostasis to treat cerebral vasospasm associated with subarachnoid hemorrhage: the Montreal Neurological Hospital protocol. NeurocritCare. 2012 Jun;16(3):354-62. https://doi.org/10.1007/s12028-012-9701-5

32. Lannes M, Zeiler F, Guichon C, Teitelbaum J. The use of milrinone in patients with delayed cerebral ischemia following subarachnoid hemorrhage: a systematic review. Can J NeurolSci. 2017 Mar;44(2):152-160. https://doi.org/10.1017/cjn.2016.316

33. Shapiro, S. Milrinone in addition to hyperdynamic therapy in the treatment of vasospasm following aneurysmal subarachnoid hemorrhage.Clinicaltrial. NCT 02712788

34. Dabus G, Nogueira RG. Currentoptions for the management of aneurysmal subarachnoid hemorrhage-induced cerebral vasospasm: a comprehensive review of the literature. InterventNeurol. 2013 Oct;2(1):30-51. https://doi.org/10.1159/000354755

35. Venkatraman A, Khawaja AM, Gupta S, Hardas S, Deveikis JP, Harrigan MR, et al. Intra-arterial vasodilators for vasospasm following aneurysmal subarachnoid hemorrhage: a meta-analysis. J NeurointervSurg. 2018 Apr;10(4):380-387. https://doi.org/10.1136/neurintsurg-2017-013128

36. Mutoh T, Kazumata K, TerasakaS, Taki Y, Suzuki A, Ishikawa T. Early intensive versus minimally invasive approach to postoperative hemodynamic management after subarachnoid hemorrhage. Stroke. 2014 May;45(5):1280-4. https://doi.org/10.1161/STROKEAHA.114.004739

37. Bilt IA, Hasan D, Vandertop WP, Wilde AA, Algra A, Visser FC, et al. Impact of cardiac complications on outcome after aneurysmal subarachnoid hemorrhage: a meta-analysis. Neurology. 2009 Feb;72(7):635-42. https://doi.org/10.1212/01.wnl.0000342471.07290.07 